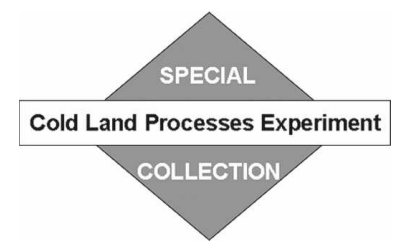

\title{
Radiative Transfer Modeling of a Coniferous Canopy Characterized by Airborne Remote Sensing
}

\author{
Richard Essery,**** Peter Bunting,* Janet Hardy, + Tim Link, \# Danny Marks, ${ }^{+}$ \\ Rae Melloh, ${ }^{+}$John Pomeroy, $\&$ Aled Rowlands,* and Nick Rutter* \\ *Institute of Geography and Earth Sciences, University of Wales, Aberystwyth, Aberystwyth, United Kingdom \\ + Cold Regions Research and Engineering Laboratory, Hanover, New Hampshire \\ \# University of Idaho, Moscow, Idaho \\ @ Northwest Watershed Research Center, Boise, Idaho \\ \& Centre for Hydrology, University of Saskatchewan, Saskatoon, Saskatchewan, Canada
}

(Manuscript received 8 January 2007, in final form 25 June 2007)

\begin{abstract}
Solar radiation beneath a forest canopy can have large spatial variations, but this is frequently neglected in radiative transfer models for large-scale applications. To explicitly model spatial variations in subcanopy radiation, maps of canopy structure are required. Aerial photography and airborne laser scanning are used to map tree locations, heights, and crown diameters for a lodgepole pine forest in Colorado as inputs to a spatially explicit radiative transfer model. Statistics of subcanopy radiation simulated by the model are compared with measurements from radiometer arrays, and scaling of spatial statistics with temporal averaging and array size is discussed. Efficient parameterizations for spatial averages and standard deviations of subcanopy radiation are developed using parameters that can be obtained from the model or hemispherical photography.
\end{abstract}

\section{Introduction}

Forest canopies strongly modify radiative fluxes reaching the underlying surface. This has important implications for hydrological and ecological processes, such as snowmelt and succession, in forested environments (Pomeroy and Dion 1996; Battaglia et al. 2002; Hardy et al. 2004). Conversely, radiation reflected and emitted from trees complicates the retrieval of forest snow properties by remote sensing (Chang et al. 1996; Klein et al. 1998). Land surface models and remote sensing algorithms, therefore, often include simple representations of radiative transfer in canopies. Variants of Beer's law or two-stream approximations are generally used (e.g., Sellers et al. 1986; Verseghy et al. 1993); these treat canopies as horizontally homogeneous tur-

** Current affiliation: School of GeoSciences, Grant Institute, University of Edinburgh, Edinburgh, United Kingdom.

Corresponding author address: Richard Essery, School of GeoSciences, Grant Institute, University of Edinburgh, Edinburgh EH9 3JW, United Kingdom.

E-mail: richard.essery@ed.ac.uk bid media and only predict the average radiation. The radiative environment beneath real canopies, however, is highly heterogeneous because of sun flecks, canopy gaps, and clearings on wide ranges of length scales. The significance of spatial heterogeneity at a particular length scale will depend on the time scale and nonlinearity of the process of interest. In snowmelt, for example, the spatial variance of shortwave radiation influences the depletion of snow-covered area (Essery and Pomeroy 2004) and longwave radiation from sunlit trunks (Faria et al. 2000; Woo and Giesbrecht 2000).

Geometrical optics and ray-tracing models have been used to simulate radiative transfer in canopies; the Radiation Transfer Model Intercomparison (Pinty et al. 2004) found that such models now compare well for homogeneous canopies but still have large discrepancies for complex heterogeneous canopies. These models are also computationally expensive and require large amounts of data on canopy structure and the optical properties of canopy elements. A great deal of work on small-scale radiative transfer through heterogeneous canopies has used statistical models that assume random tree distributions or spatially explicit models with randomly generated tree distributions 
(e.g., Satterlund 1983; Li et al. 1995; Yang et al. 2001; Song and Band 2004). Fewer studies have used real structural data from natural or managed forests (e.g., Courbaud et al. 2003; Stadt et al. 2005). Hemispherical photography provides a method for relatively rapid gathering of canopy structure information in the field (Rich 1990), and several software packages have been developed for modeling forest light environments from hemispherical photography, but it is difficult to apply this to large areas or inaccessible locations. Airborne remote sensing enables collection of large amounts of canopy data but has not yet been widely used in radiation modeling.

The aims of this paper are to demonstrate the use of remote sensing in mapping of forest stand characteristics for radiative transfer modeling, to develop a model that uses stand maps to simulate patterns of solar radiation beneath the canopy, to evaluate the model in comparison with ground-based measurements, to investigate the spatial and temporal scaling of subcanopy radiation statistics, and to develop parameterizations of those statistics. Data from aerial photography and lidar (light detection and ranging) scanning of a coniferous forest with areas of varying canopy density and uniformity are used, as described in the next section. Crowns are delineated in the photograph to map the location and crown diameter of each tree, and tree heights are assigned from lidar elevation returns. A spatially explicit model that uses these data to simulate transmission of solar radiation to the forest floor is developed in section 3; the complex pattern of transmission through canopy gaps is explicitly represented, but, for numerical efficiency and to minimize the number of parameters required, a very simple scheme is used for transmission through crowns. Model results are compared with ground-based hemispherical photography and distributed measurements of radiation under the canopy on clear and cloudy days in section 4 . Because the spatially explicit model is too computationally expensive to run for large areas or long times, efficient parameterizations of radiation statistics are sought in section 5. Finally, section 6 presents conclusions and discusses future plans.

\section{Site and data description}

The Cold Land Processes Experiment (CLPX) was conducted in Colorado during the winters of 2002 and 2003. Data used in this study were obtained at the Local Scale Observation Site (LSOS; Hardy et al. 2004, 2007, manuscript submitted to J. Hydrometeor.), which was within the U.S. Forest Service Fraser Experimental Forest $\left[39.9^{\circ} \mathrm{N}, 105.9^{\circ} \mathrm{W}, 2780 \mathrm{~m}\right.$ above sea level (a)

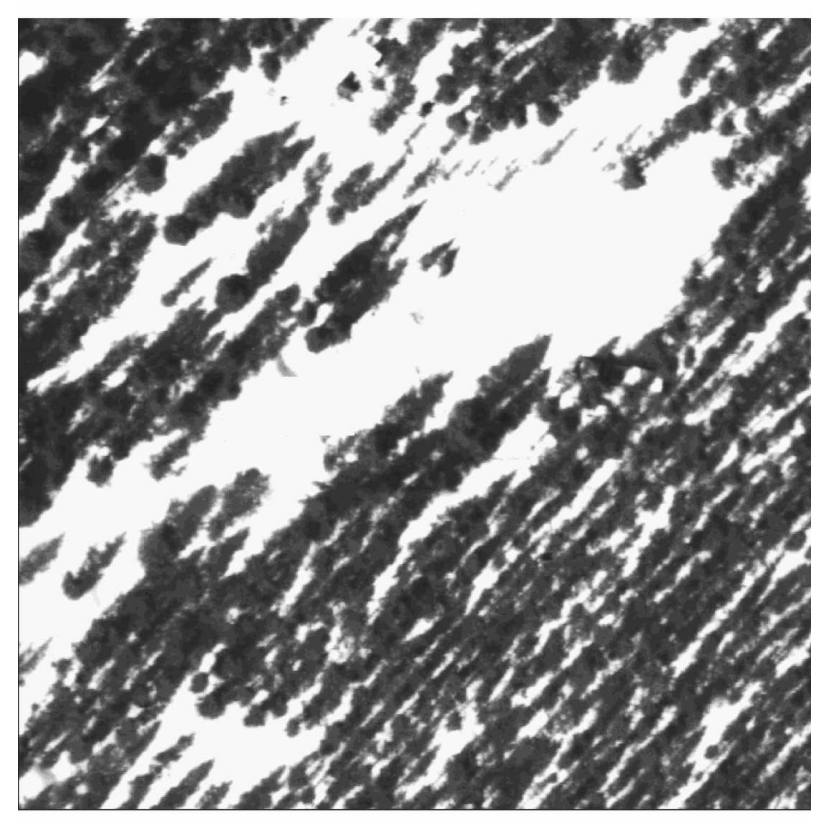

(b)

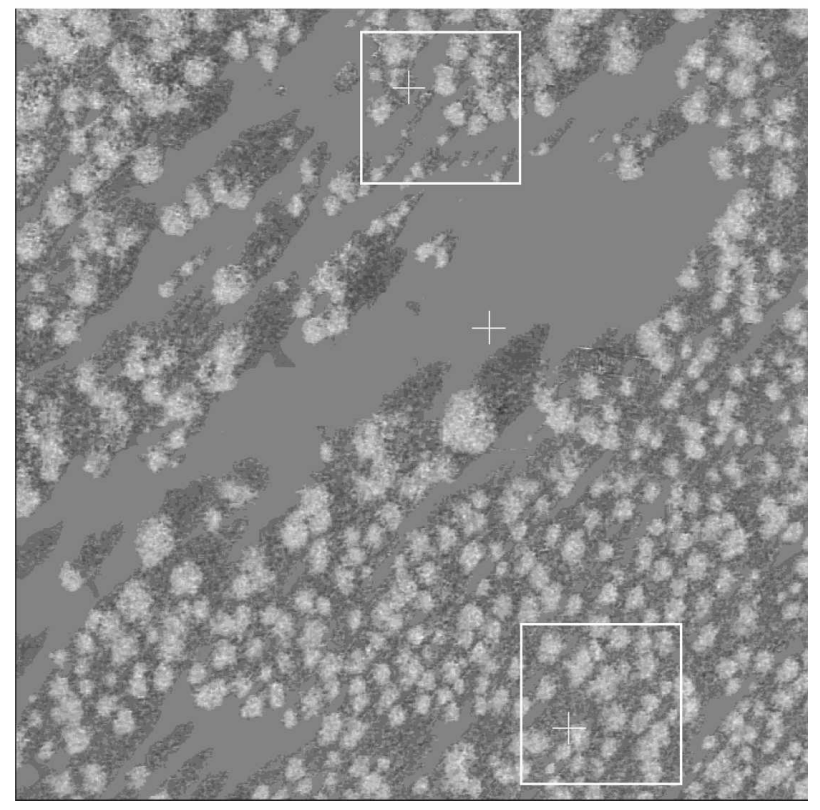

FIG. 1. (a) Aerial color infrared orthophotograph (shown in black and white) of the study site. The area shown is $100 \mathrm{~m} \times 100$ $\mathrm{m}$, with north at the top. (b) Normalized difference between nearinfrared and red bands of the photograph. Boxes show the areas in which radiometer arrays were deployed, and crosses show the locations at which the hemispherical photographs in Fig. 4 were taken.

(ASL)] and is shown by the color infrared orthophotograph in Fig. 1a. A clearing separates two stands of contrasting structure. To the south of the clearing, there is a uniform lodgepole pine plantation; from manual 

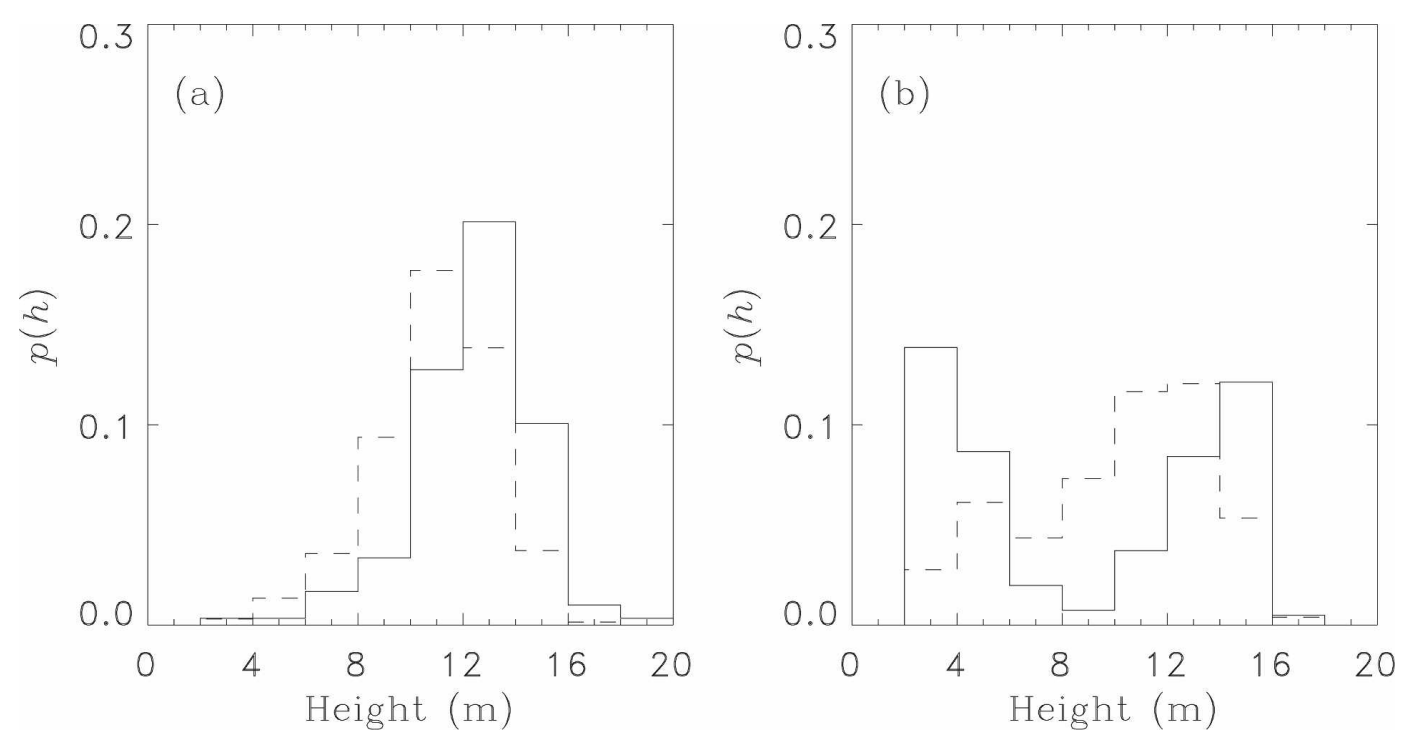

FIG. 2. Histograms of tree heights from ground surveys (solid lines) and lidar elevations (dashed lines) for the (a) uniform and (b) discontinuous stands.

surveying, the trees have an average height of $12.6 \mathrm{~m}$ with standard deviation $2.4 \mathrm{~m}$. To the north of the clearing, there is a discontinuous mixed-age stand, predominantly of lodgepole pine but with some subalpine fir and Engelmann spruce; the average tree height is $9.3 \mathrm{~m}$ and the standard deviation is $4.9 \mathrm{~m}$. The histograms in Fig. 2 show that the tree heights have an approximately normal distribution for the uniform stand but a bimodal distribution of short and tall trees for the discontinuous stand.

Solar radiation was measured beneath the uniform

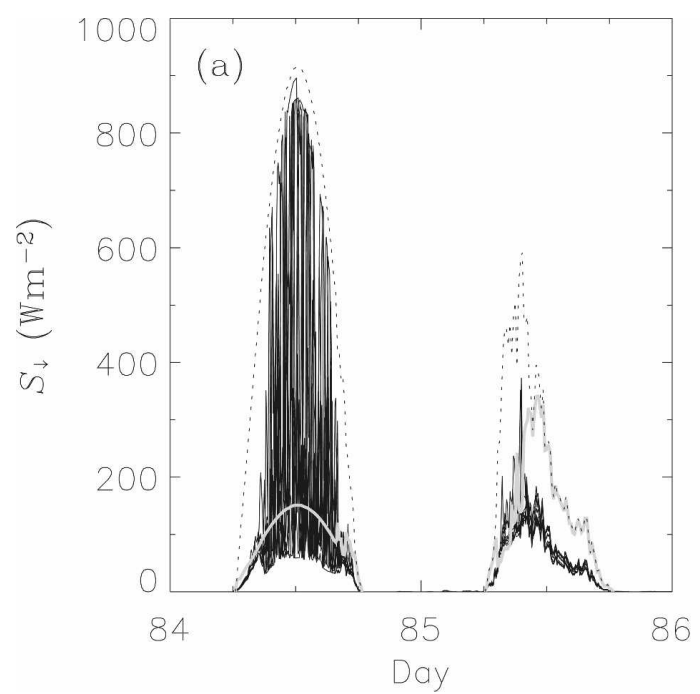

and discontinuous canopies within the areas shown by boxes in Fig. 1b with two radiometer arrays (Link et al. 2004; Pomeroy et al. 2008, hereafter POM), each consisting of 10 Eppley pyranometers $(0.3-3-\mu \mathrm{m}$ wavelength, $160^{\circ}$ sky view, $5 \%$ accuracy). Figure 3 shows 5 -min averages of 10-s measurements from each array for days 84 and 85 (24 and 25 March) in 2003. Also shown is the above-canopy radiation measured by a radiometer mounted at a height of $18 \mathrm{~m}$ on a mast in a clearing $200 \mathrm{~m}$ to the southwest of the study area. Day 84 was clear, with strong direct illumination in open

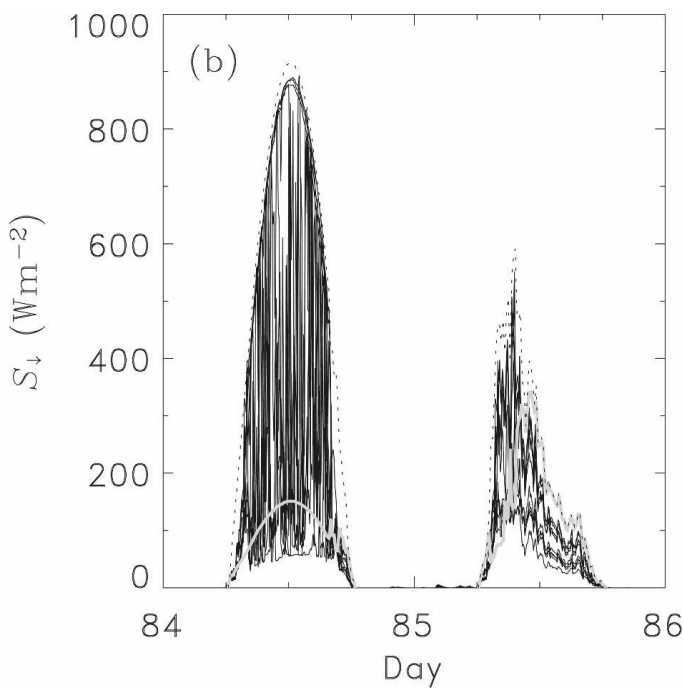

FIG. 3. Solar radiation measured by 10-radiometer arrays in the (a) uniform and (b) discontinuous stands. Dotted lines show incoming radiation measured above the canopy, and gray lines show diffuse radiation estimated by the method described in section 3 . 


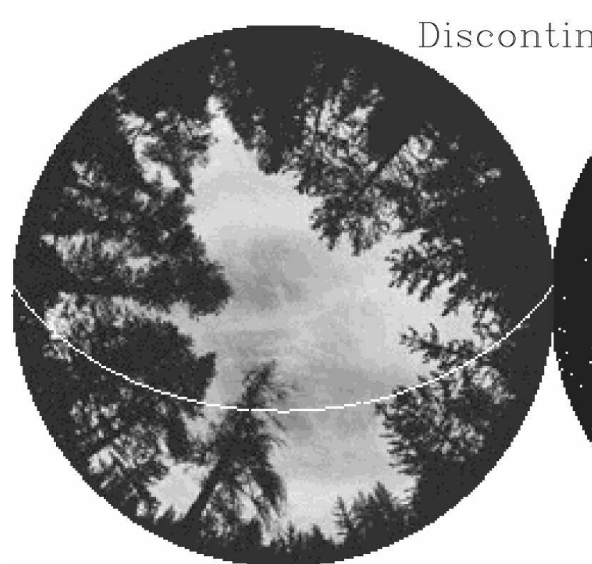

inuous

\section{Simulation}
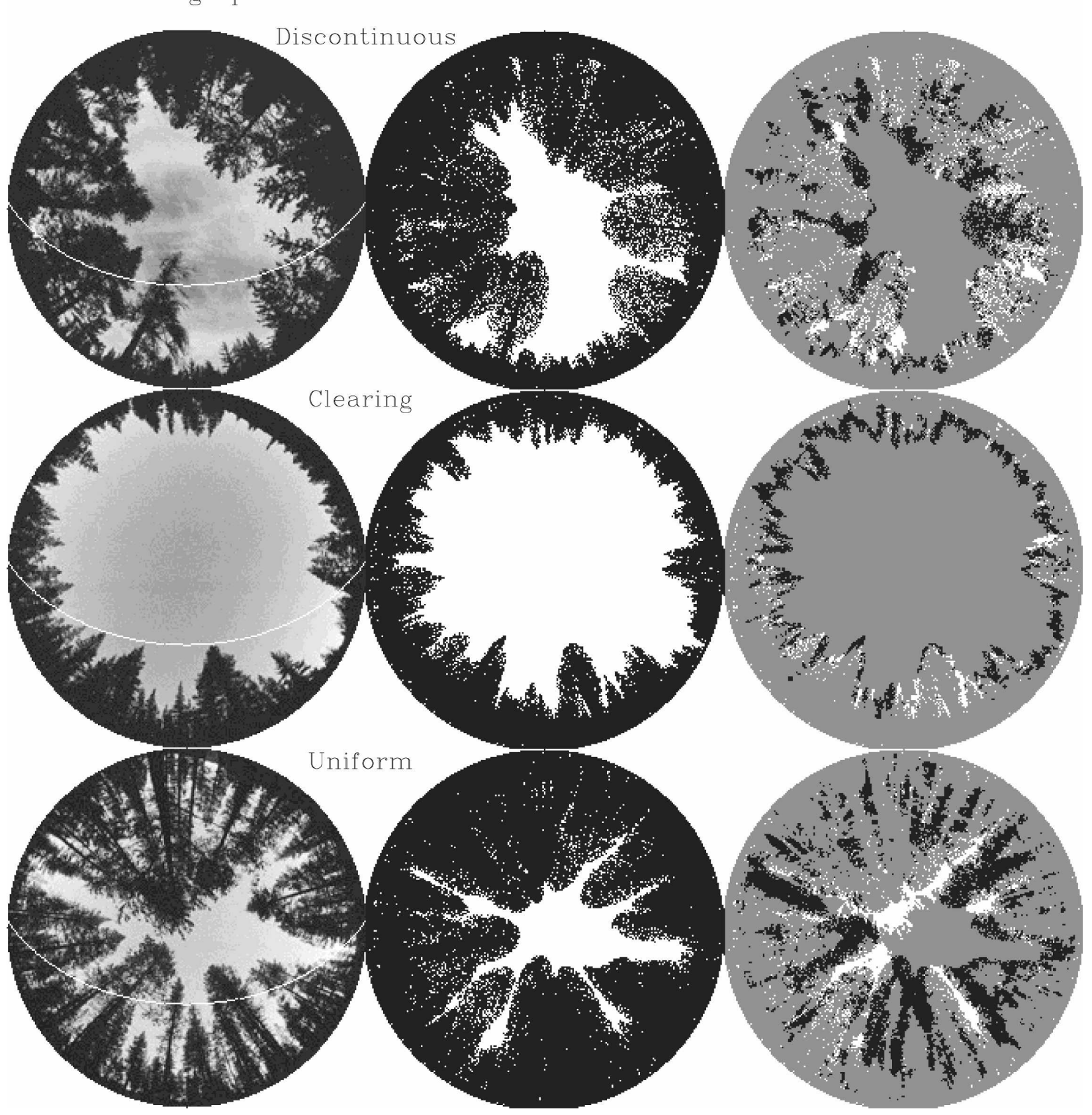

FIG. 4. Observed and simulated hemispherical sky view for the points marked by crosses in Fig. 1b. White lines on the photographs show the sun track for 24 Mar. Images on the right show differences between sky masks obtained from the photographs and the model, with white pixels where the model is more open than observed, black pixels where it is more closed, and gray pixels where the two agree.

areas, whereas day 85 was overcast. Averages and standard deviations across the arrays are larger for the discontinuous stand than the uniform stand and larger for the clear day than the overcast day.

Hemispherical photographs were taken at points on a staggered 20-m grid (Melloh et al. 2003); example photographs from the discontinuous stand, the clearing, and the uniform stand at the points marked by crosses in Fig. $1 \mathrm{~b}$ are shown in Fig. 4, overlain with the sun track for day 84 . These photographs were used by Melloh et al. (2003) to study gap fraction distributions and by Hardy et al. (2004) to model transmission of solar radiation through the canopy with the Gap Light Analyzer software package (Frazer et al. 1999). 
Before delineation of the orthophotograph to identify individual tree crowns, forest and nonforest classes have to be discriminated. Because the radiative properties of snow and vegetation differ strongly, the normalized difference vegetation index (NDVI) between the near-infrared (NIR) and red bands of the photograph,

$$
\mathrm{NDVI}=\frac{\mathrm{NIR}-\mathrm{RED}}{\mathrm{NIR}+\mathrm{RED}}
$$

is found to give good discrimination between tree crowns and areas of sunlit or shaded snow, as shown in Fig. 1b. By varying the threshold for discrimination across the image by an image segmentation procedure using the local standard deviation, smaller crowns that are increasingly confused with snow because of reflection from ground beneath the canopy can be identified while providing good definition of the forest boundary in denser areas.

Four main techniques have been developed for automatic crown delineation in images: valley following (Gougeon 1995), pattern matching (Pollock 1996), crown centroid identification (Culvenor 2002), and wavelet analysis (Strand et al. 2006). The crown centroid method used here is described by Bunting and Lucas (2006). Local maxima in NDVI are identified as crown centers and expanded to minima to form crown edges. A classification procedure identifies and removes objects identified as complete crowns, and the remaining objects are further split until all objects are classified as crowns or a maximum number of iterations has been reached. Finally, correction procedures to identify and merge oversplit crowns are used to produce the final result. The method has been particularly successful in identifying crowns of varying size and shape, achieving accuracies of $70 \%$ for complex mixed species forests in Australia. Although the LSOS stands are relatively homogeneous in crown shape and size, many crowns overlap or have interlocking branch structures. Compared with many other algorithms that leave a pixel-wide gap between crowns, it is believed that the algorithm used here is more robust for overlapping crowns.

The CLPX lidar data have an approximately $1.5-\mathrm{m}$ horizontal spacing and were supplied filtered into ground and vegetation elevation returns (Miller 2003). The ground returns were triangulated and interpolated onto a regular grid to form a digital elevation model, but the site is nearly level; surface elevations for the area shown in Fig. 1 only increase by $2 \mathrm{~m}$ from the southwest corner to the northeast corner. Surface heights were subtracted from the vegetation returns to

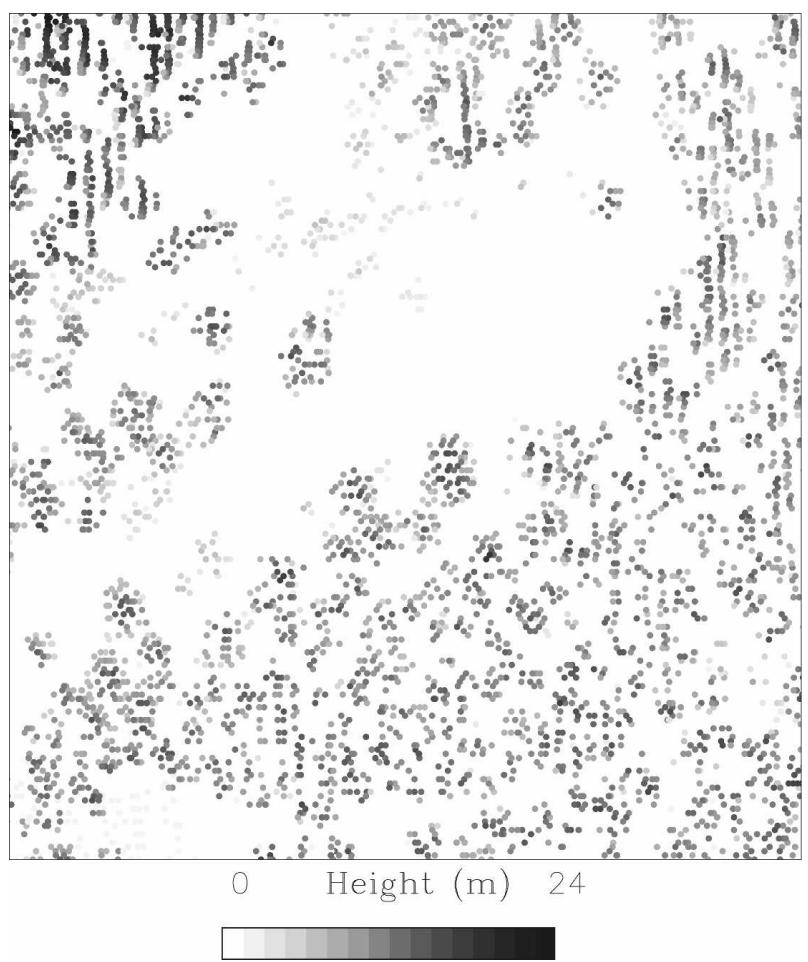

FIG. 5. Lidar vegetation heights for the area shown in Fig. 1.

generate the map of vegetation heights shown in Fig. 5. Histograms of lidar heights for trees in the manually mapped areas of the uniform and discontinuous stands are plotted in Fig. 2. Compared with the ground survey results, the average tree height is underestimated in the uniform stand and the short trees in the discontinuous stand are underrepresented, but the histogram shapes are similar. Lidar can miss small trees and can underestimate the height of a tree if the highest point is not sampled or if the laser penetrates some distance into the canopy before being reflected; methods have been developed to correct for this (Magnussen et al. 1999), but the lidar elevation data are used here without adjustment.

\section{Model development}

The model developed here represents individual trees as geometric primitives to allow analytic calculations of pathlengths for radiation beams passing through the canopy. This approach has been used in several other models (e.g., Satterlund 1983; Li et al. 1995; Song and Band 2004), but the actual implementation used here is based on Price (1997). Trees are assumed to have ellipsoidal canopies and cylindrical trunks; the pathlength calculation for a single tree is shown schematically in Fig. 6 . A ray from point $\left(x_{s}, y_{s}\right)$ 


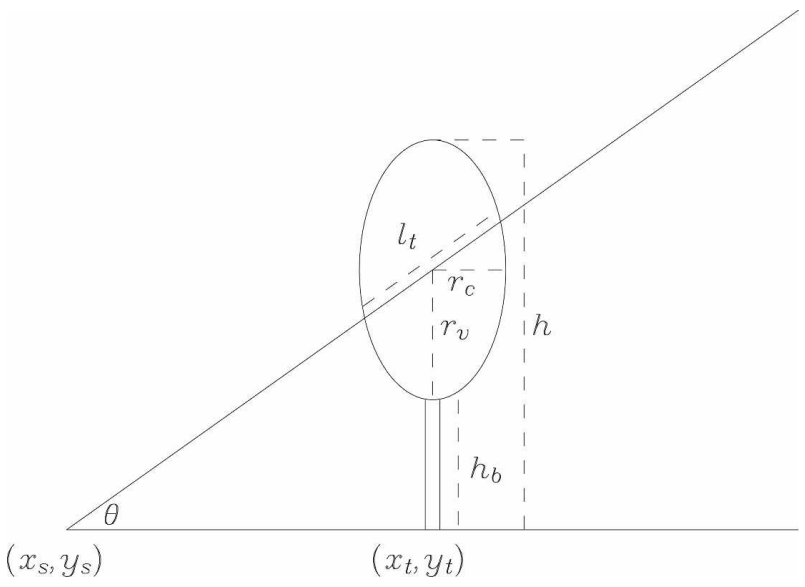

FIG. 6. Schematic illustration of the pathlength $l_{t}$ for an ellipsoidal tree crown of radius $r_{c}$, height $h$, and base $h_{b}$.

on the surface in the direction of the sun is defined by the parametric vector

$$
r(l)=\left(x_{s}+l \cos \theta \sin \phi, y_{s}-l \cos \theta \cos \phi, l \sin \theta\right),
$$

where $l$ is the distance from the surface, $\theta$ is the solar elevation, and $\phi$ is the solar azimuth, measured anticlockwise from south by convention. For a tree at point $\left(x_{t}, y_{t}\right)$ with height $h$, crown radius $r_{c}$, and canopy base height $h_{b}$, the crown surface is defined by the solutions of the equation

$$
\frac{\left(x-x_{t}\right)^{2}+\left(y-y_{t}\right)^{2}}{r_{c}^{2}}+\frac{\left(z-h+r_{v}\right)^{2}}{r_{v}^{2}}=1,
$$

where $r_{v}=\left(h-h_{b}\right) / 2$ is the vertical half axis of the crown ellipsoid. Substituting Eq. (2) into Eq. (3) to find the points at which the ray intersects the crown gives a quadratic equation:

$$
a l^{2}+b l+c=0,
$$

where

$$
\begin{aligned}
& a=\frac{\cos ^{2} \theta}{r_{c}^{2}}+\frac{\sin ^{2} \theta}{r_{v}^{2}}, \\
& b=\frac{2 \cos \theta\left[\left(x_{s}-x_{t}\right) \sin \phi-\left(y_{s}-y_{t}\right) \cos \phi\right]}{r_{c}^{2}}+\frac{2 \sin \theta\left(r_{v}-h\right)}{r_{v}^{2}},
\end{aligned}
$$

and

$$
c=\frac{\left(x_{s}-x_{t}\right)^{2}+\left(y_{s}-y_{t}\right)^{2}}{r_{c}^{2}}+\frac{\left(r_{v}-h\right)^{2}}{r_{v}^{2}}-1 .
$$

The ray intersects the crown if and only if $D=b^{2}-$ $4 a c>0$. The pathlength through the crown is then $l_{t}=$ $a^{-1} D^{1 / 2}$, and the total pathlength is found by summing the pathlengths through all crowns that the ray intersects.

Points in the shade of a cylindrical trunk can be found by replacing $r_{c}$ with trunk radius $r_{t}$ and deleting terms involving $r_{v}$ in Eqs. (5)-(7), rejecting solutions with intersection heights lying outside the range $[0, h]$. Trunks and canopy elements are assumed to be opaque, and multiple scattering within the canopy or between the canopy and the ground are neglected as a first approximation, but light can pass through gaps within the canopy. Following Nilson (1971), the probability that a beam will pass through the canopy without interception is

$$
p_{t}=\exp \left[-G(\theta) \Sigma \lambda l_{t}\right]
$$

where $G$ is a projection function determined by the orientation of canopy elements, $\lambda$ is the effective foliage area volume density, and the summation is over all crowns that the beam intersects. Random orientations are assumed, for which $G=0.5$. Foliage area and crown volume could, in principal, be measured by lidar, but this requires a relationship with the density of ground returns from under the canopy to be developed (Riaño et al. 2004). Ground measurements are used here instead. Kaufmann et al. (1982) measured leaf areas for lodgepole pine at Fraser and obtained an allometric relationship with tree height; dividing by the volume of an ellipsoidal crown gives

$$
\lambda=\frac{2.925 h^{2}}{4 \pi r_{v} r_{c}^{2}} .
$$

The effective value of $\lambda$ in Eq. (8) is increased by woody material in the canopy but decreased by clumping of canopy elements (Chen et al. 1997); lacking sufficient information for lodgepole pine, Eq. (9) is used without adjustment.

Crown radii and centers are found by delineation of the orthophotograph, as described above. The tree height is taken to be given by the highest lidar elevation within the crown profile, without correction. Trunk radii and canopy base heights cannot be directly determined from the above-canopy measurements. Instead, allometric relationships obtained from manual measurements of 198 trees in the LSOS area are used. Regression against tree height gives $r_{t}=0.01 h^{1.15}\left(r^{2}=\right.$ 0.81 , rms error $0.04 \mathrm{~m})$ and $h_{b}=0.02 h^{1.86}\left(r^{2}=0.64\right.$, rms error $1.59 \mathrm{~m}$ ).

Because rays are traced from the ground surface upward in the direction of the sun, sloping surfaces can easily be included in an extension of the model; topography has important influences on radiative transfer through canopies (Melloh et al. 2003; Liu et al. 2004), 
but the site considered here is level. The ellipsoidal crowns can be replaced by conical or parabolic canopies; more general geometric shapes (Sheng et al. 2001) or realistic crown shape models could also be used, but analytic solutions for the pathlength are then no longer available and the computational expense is greatly increased.

To generate sun flecks within a tree shadow, rather than a homogeneous shadow, $p_{t}$ is taken to be a random variable with values of 0 or 1 and average given by Eq. (8). In general, the downward radiation at a point $(x, y)$ on the surface is

$$
\begin{aligned}
S_{\downarrow}(x, y)= & \frac{1}{\pi} \int_{0}^{2 \pi} d \phi \int_{\theta_{h}(\phi)}^{\pi / 2} S_{0}(\theta, \phi) p_{t}(x, y ; \theta, \phi) \\
& \times \sin \theta \cos \theta d \theta,
\end{aligned}
$$

where $S_{0}(\theta, \phi)$ is the radiation from elevation $\theta$ and azimuth $\phi$ above the canopy, $p_{t}(x, y ; \theta, \phi)$ is the probability of transmission through the canopy from that direction, and $\theta_{h}(\phi)$ is the elevation angle of the horizon, which can be calculated from the DEM (Dozier and Frew 1990). Two assumptions are made to simplify the evaluation of Eq. (10): taking diffuse radiation from the sky to be isotropic and treating the sun as a point source gives

$$
S_{\downarrow}(x, y)=v(x, y) S_{\mathrm{dif}}+p_{t}(x, y ; \theta, \phi) S_{\mathrm{dir}},
$$

where $S_{\text {dif }}$ is the diffuse component of the abovecanopy radiation, $S_{\mathrm{dir}}$ is the direct component, and

$$
v(x, y)=\frac{1}{\pi} \int_{0}^{2 \pi} d \phi \int_{\theta_{h}(\phi)}^{\pi / 2} p_{t}(x, y ; \theta, \phi) \sin \theta \cos \theta d \theta
$$

is the sky-view factor, which is evaluated by numerical integration with values of $p_{t}$ provided by the model.

Separate measurements of diffuse radiation above the canopy were not made, so it is estimated by the empirical method of Erbs et al. (1982). First, atmospheric transmissivity is found by dividing the abovecanopy radiation by the radiation at the top of the atmosphere, giving

$$
k_{t}=\frac{S_{0}}{I_{0} \sin \theta},
$$

where $I_{0}=1367 \mathrm{~W} \mathrm{~m}^{-2}$ is the solar constant. The diffuse radiation is then estimated as $S_{\mathrm{dif}}=d S_{0}$, with

$$
d=\left\{\begin{array}{cc}
1-0.09 k_{t} & k_{t}<0.22 \\
0.95-0.16 k_{t}+4.39 k_{t}^{2}-16.64 k_{t}^{3}+12.34 k_{t}^{4} & 0.22<k_{t}<0.8 \\
0.165 & k_{t}>0.8
\end{array}\right.
$$

Direct radiation is the remainder $S_{\mathrm{dir}}=S_{0}-S_{\mathrm{dif}}$.

\section{Results}

Figure 7 shows simulations of tree shadows for the time at which the orthophotograph in Fig. 1 was taken [1400 local standard time (LST) 8 April] and sky-view factors, which are independent of time. There is good subjective similarity between Figs. 1a and 7a, although the shadows of isolated trees show that the ellipsoidal crowns in the simulation are a little too rounded. Close inspection suggests that the delineation tends to oversegment the canopy in denser areas, giving too many trees and too great a canopy coverage.

The sky view in Fig. 7b shows both small-scale variability within stands and the large-scale structure of stands and gaps. Hemispherical sky views can be simulated by dividing the sky hemisphere into azimuth and elevation cells and blanking out cells that are obstructed by the canopy according to the model; Melloh et al. (2003) previously did this for LSOS using manual inventory maps and a tree model. Simulated hemi- spherical images and differences between sky masks obtained from simulations and photographs were included in Fig. 4 for comparison with the photographs. There are some evident errors in tree positions, heights, and shapes, and the gaps between trees are too small in the uniform stand, but the broad patterns are in good agreement. Simulated sky-view factors are scattered against values obtained from the full set of hemispherical photographs in Fig. 8; error bars for uncertainty in the registration of measurement points within the model were estimated by displacing the simulation points by $2 \mathrm{~m}$ in each of the cardinal directions, and error bars for uncertainty in creating sky masks from the photographs were estimated by changing the threshold brightness by $\pm 10 \%$. The overall agreement is good, with bias -0.02 , rms error 0.09 , and $r^{2}=0.8$. Most of the points at which the sky view is underestimated are in the uniform stand.

The model was run on a 1-m grid for days 84 and 85 with a 5-min time step, and statistics were calculated for the $20 \mathrm{~m} \times 20 \mathrm{~m}$ areas within which the radiometer arrays were deployed. The measurements sample too 
(a)

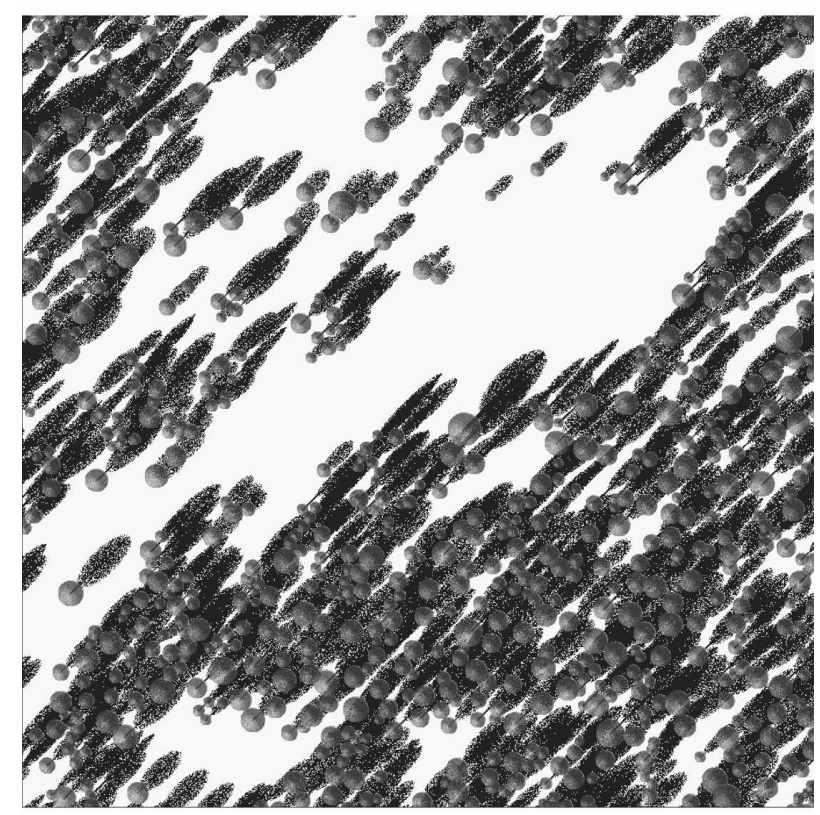

(b)
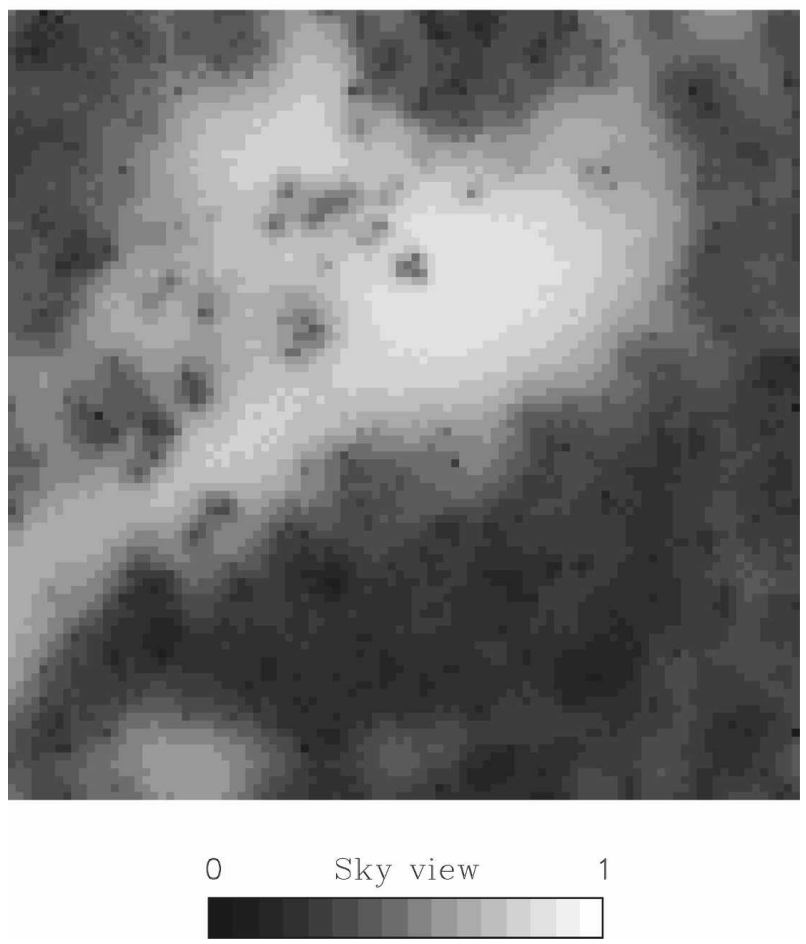

FIG. 7. (a) Simulated tree shadows and (b) sky-view factors for the area shown in Fig. 1.

few points to calculate histograms of subcanopy radiation, but they can be used to calculate cumulative distributions giving fractions of the surface receiving less than some specified amount of radiation. Cumulative

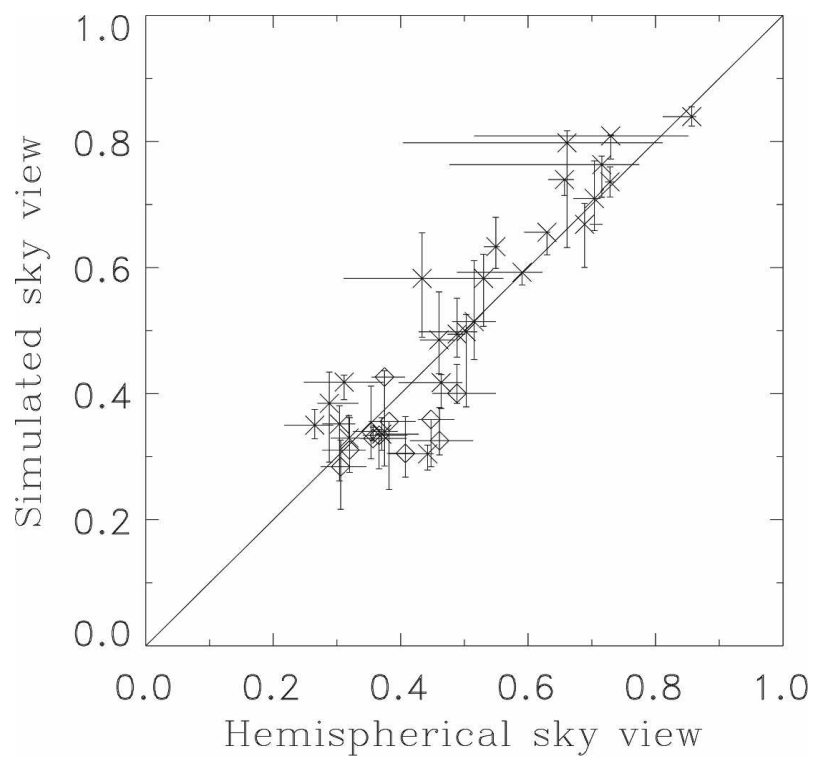

FIG. 8. Sky-view factors simulated by the model and calculated from hemispherical photographs; crosses show points in open areas and the discontinuous stand, and diamonds show points in the uniform stand. Vertical bars give the range of values obtained by displacing the simulation points by $2 \mathrm{~m}$ in each of the cardinal directions, and horizontal bars give the range of values obtained by varying the brightness threshold applied to the photographs by $\pm 10 \%$.

distributions at noon on clear-sky day 84 are shown in Fig. 9 for simulations and measurements in the uniform and discontinuous stands. The distributions can be expected to be highly bimodal on clear days, with some radiometers in direct sun and others shaded, and this gives sills that are evident in the measured and simulated distributions. The height of the sill corresponds to the fraction of the forest floor in shade, which is simulated as $75 \%$ for the uniform stand and $55 \%$ for the discontinuous stand. Because of the bimodal nature of the distributions, standard error estimates based on normal distributions are inappropriate. Instead, 90\% confidence limits for the measured distributions were estimated by generating a large number of 10-point random samples from the simulated radiation distributions; these are shown by dotted lines on Fig. 9. The agreement between the simulation and measurements is good for the discontinuous stand, but the simulation underestimates the radiation in both sunlit and shaded fractions of the uniform stand, consistent with the underestimate of sky view noted above. Although it might be expected from the conclusions of Pinty et al. (2004) that radiation would be harder to simulate in the discontinuous stand, it turns out that it is harder to delineate the tree crowns accurately in the denser, more uniform parts of the canopy. 

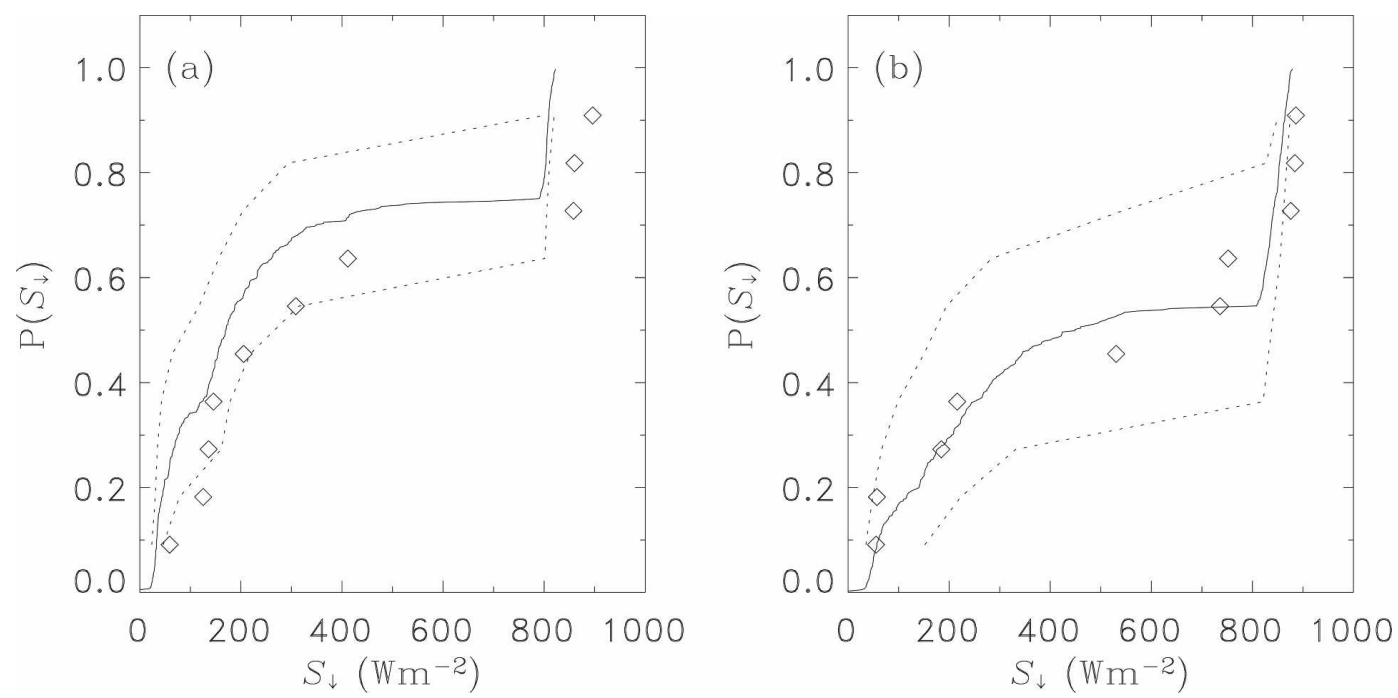

FIG. 9. Cumulative distributions of subcanopy radiation at noon on clear-sky day 84 for the (a) uniform and (b) discontinuous stands from array measurements (diamonds) and simulations (solid lines). Dotted lines show simulated $90 \%$ confidence intervals for random 10-point samples.

Averages and standard deviations from the array measurements are compared with simulations in Fig. 10 , and simulated $90 \%$ confidence intervals on the statistics for random 10-point samples are also shown. The average is underestimated for the discontinuous stand and the standard deviation is overestimated for the uniform stand on day 85 before noon, but the measurements generally lie within the confidence intervals on the simulation results. The simulated statistics are smoother than the measured statistics because some of the spatial variability appears as temporal variability when a moving pattern with high spatial variability is sampled. Confidence intervals on 5-min averages for 10-radiometer arrays turn out to be quite broad as a result. Figure 11 shows how simulated confidence intervals on sample averages tighten with increasing sample size for 5-min and daily averages. Both scale with $N^{-1 / 2}$ as expected, but many more radiometers are required to estimate 5-min averages with the same confidence as daily averages.

Because patches of sunlight on the forest floor move during the day, it can be expected that the standard deviation of solar radiation will decrease if the averaging period is increased. For the same sites considered here but a different period, POM investigated how measured coefficients of variation $(\mathrm{CV})$ scale with averaging period. Figure 12 shows $\mathrm{CVs}$ calculated from array data averaged over periods from $5 \min$ to $12 \mathrm{~h}$ centered on noon of clear-sky day 84. As noted by $\mathrm{POM}$, the $\mathrm{CV}$ is greater for the uniform stand than the discontinuous stand over short intervals but decreases more rapidly as the averaging period is increased.
Simulated CVs show the same qualitative behaviors, but the decrease in $\mathrm{CV}$ for the uniform stand is less dramatic in the simulation than in the measurements; this is because the average is lower and the standard deviation is higher in the simulated daily radiation. CVs for overcast days (not shown) have much less dependence on temporal averaging.

Standard deviations are plotted against averages in Fig. 13 for clear and overcast conditions, discriminated here as periods with diffuse radiation fraction $d<0.17$ and $d>0.9$, respectively. POM found the measured standard deviation to be a linear function of average radiation under overcast conditions, and the simulation produces the same behavior. For clear conditions, a nonlinear relationship is observed and simulated, with decreasing rates of change in standard deviations at higher averages. The simulation for the discontinuous stand shows hysteresis because of the greater openness to the southeast of this area than to the southwest.

\section{Parameterization of radiation statistics}

From Eq. (11), the average and variance of subcanopy radiation are

$$
\bar{S}_{\downarrow}=\bar{v} S_{\mathrm{dif}}+\bar{p}_{t} S_{\mathrm{dir}}
$$

and

$$
\sigma_{S}^{2}=\sigma_{v}^{2} S_{\mathrm{dif}}^{2}+\sigma_{p}^{2} S_{\mathrm{dir}}^{2}+2 \operatorname{cov}\left(v, p_{t}\right) S_{\mathrm{dif}} S_{\mathrm{dir}},
$$

where overbars denote spatial averages, $\sigma_{p}^{2}=\bar{p}_{t}\left(1-\bar{p}_{t}\right)$ is the variance of $p_{t}, \sigma_{v}^{2}$ is the variance of $v$, and 

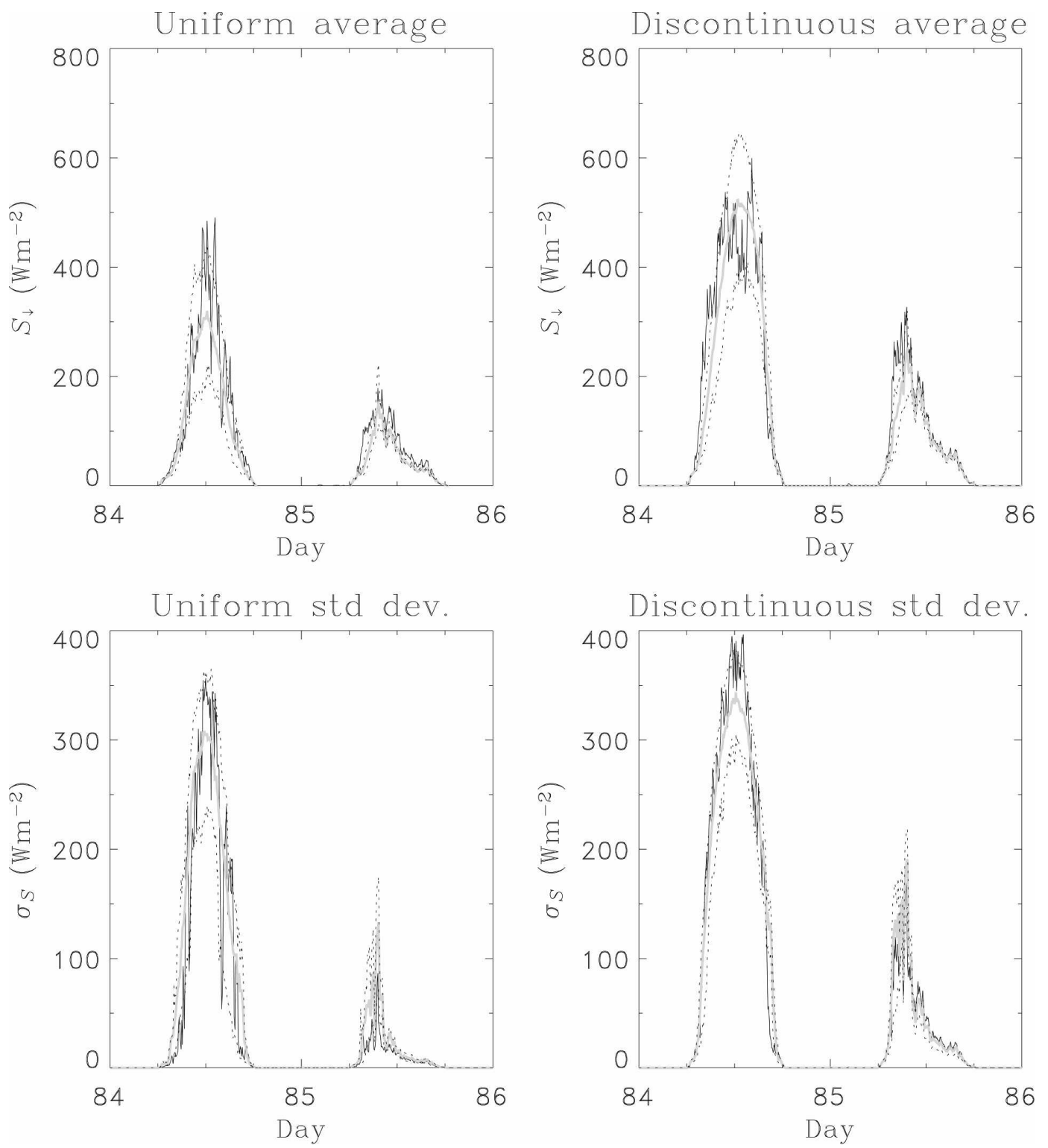

FIG. 10. (top) Averages and (bottom) std dev of subcanopy solar radiation from array measurements (black lines) and simulations (gray lines) for (left) uniform and (right) discontinuous stands. Dotted lines show simulated $90 \%$ confidence intervals for random 10-point samples.

$\operatorname{cov}\left(v, p_{t}\right)$ is their covariance. Equation (12) gives the covariance as an integral over the autocorrelation of the transmissivity. This autocorrelation is an important quantity related to the bidirectional reflectance of a forest scene and warrants further investigation, but neglecting covariance between sky view and transmission simplifies the parameterization of Eq. (16). The coefficient of variation is then given by

$$
\mathrm{CV}=\frac{\left[\sigma_{v}^{2} d+\bar{p}_{t}\left(1-\bar{p}_{t}\right)(1-d)\right]^{1 / 2}}{\bar{v} d+\bar{p}_{t}(1-d)}
$$

where $d$ is the diffuse fraction from Eq. (14) or measurements. This predicts that the $\mathrm{CV}$ should have a constant value of $\sigma_{v} / \bar{v}$ for overcast conditions and decrease with increasing transmission for clear conditions, as noted in the previous section for both observations and simulations.

A parameterization is required for $\bar{p}_{t}$. With the assumption of opaque canopy elements, $\bar{p}_{t}$ for a particular elevation is equal to the gap fraction at that elevation, which can be measured from real or simulated hemispherical images. Azimuthal averages of simulated gap 

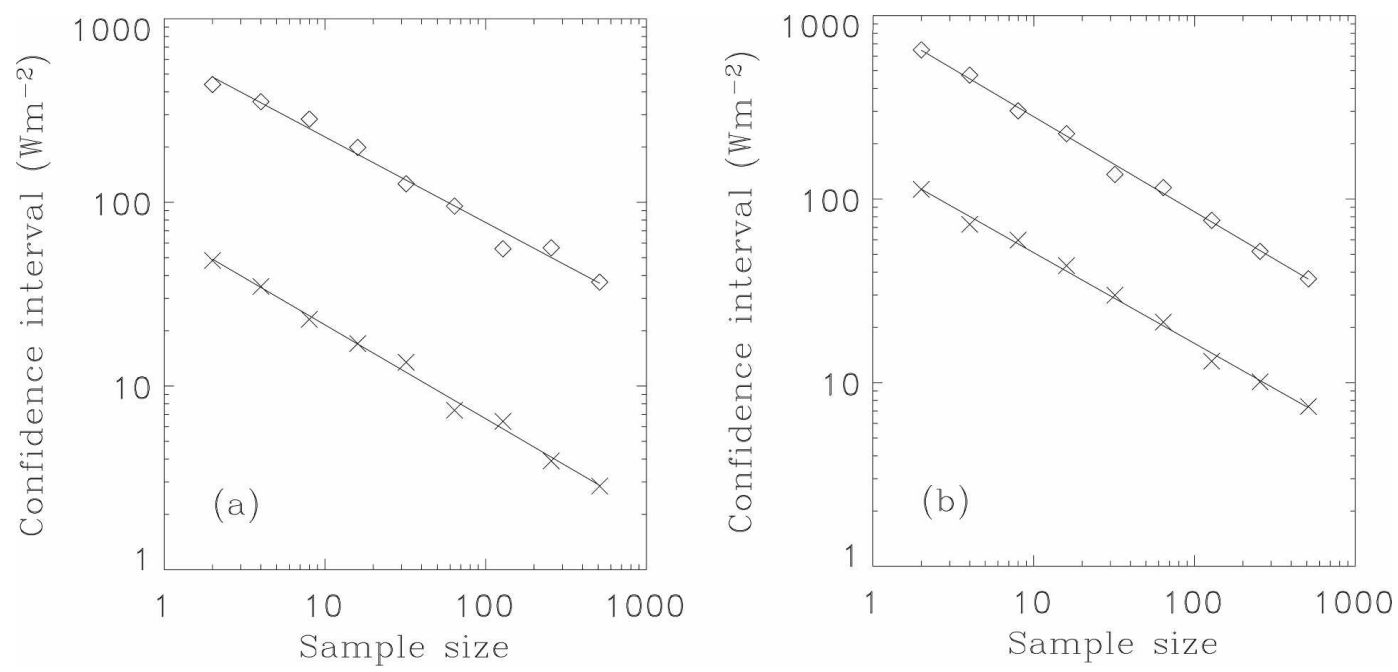

FIG. 11. Simulated $90 \%$ confidence intervals on estimates of spatial averages for varying sample sizes and 5-min (diamonds) or daily (crosses) temporal averaging for the (a) uniform and (b) discontinuous stands. The fitted lines are $N^{-1 / 2}$ power laws.

fractions for the uniform stand, the discontinuous stand, and a $10 \mathrm{~m} \times 10 \mathrm{~m}$ area in the middle of the clearing are shown in Fig. 14. It is found that Beer's law,

$$
\bar{p}_{t}(\theta)=\exp \left[-\frac{g(\theta)}{\cos \theta}\right]
$$

with the apparent foliage projection function $g$ given by the parametric form

$$
g(\theta)=a+b \sin \theta+c \cos \theta
$$

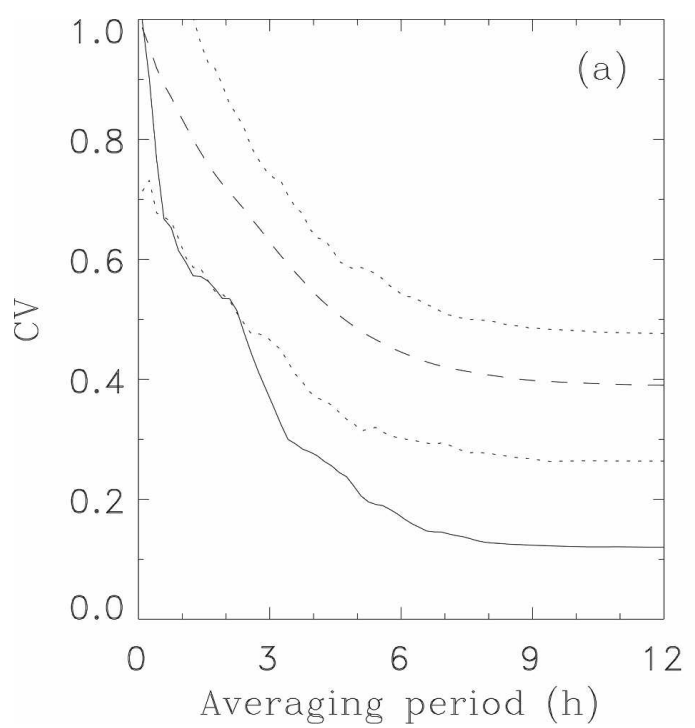

fits the modeled gap fractions closely for the parameter values given in Table 1 . The value of $\bar{p}_{t}$ at $\theta=90^{\circ}$ gives the canopy openness; for the clearing, Eq. (18) has to be limited to give $\bar{p}_{t} \leq 1$.

Substituting Eq. (18) into Eq. (12), the sky-view statistics could be expressed in terms of elliptic integrals (Nijssen and Lettenmaier 1999). The simpler approach of taking values from the simulation, given in Table 1 , is used here. Equations (15) and (16) are compared with observations and simulations in Fig. 13. The parameterizations match the statistics of the spatially ex-

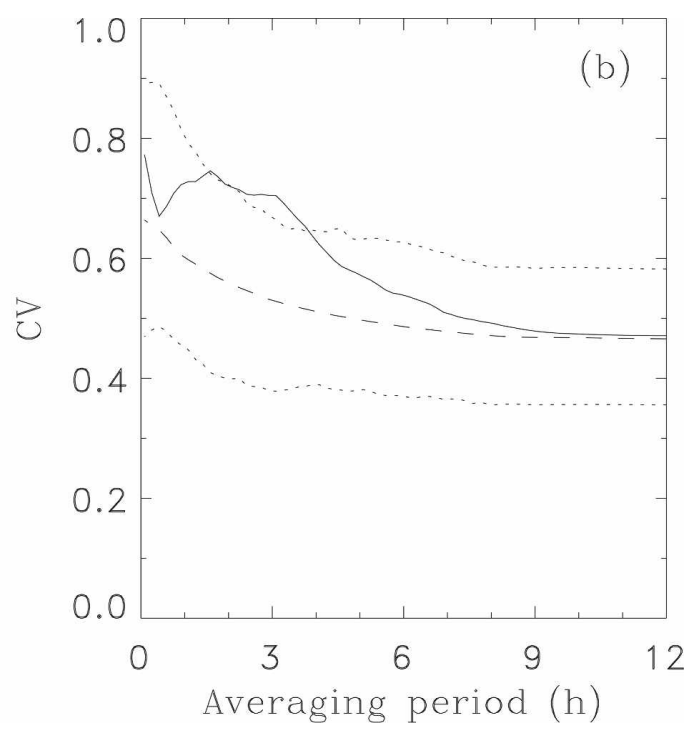

FIG. 12. Coefficients of variation in subcanopy radiation averaged over varying periods centered on noon of clear-sky-day 84 for the (a) uniform and (b) discontinuous stands from array measurements (solid lines) and simulations (dashed lines). Dotted lines show simulated 90\% confidence intervals for random 10-point samples. 

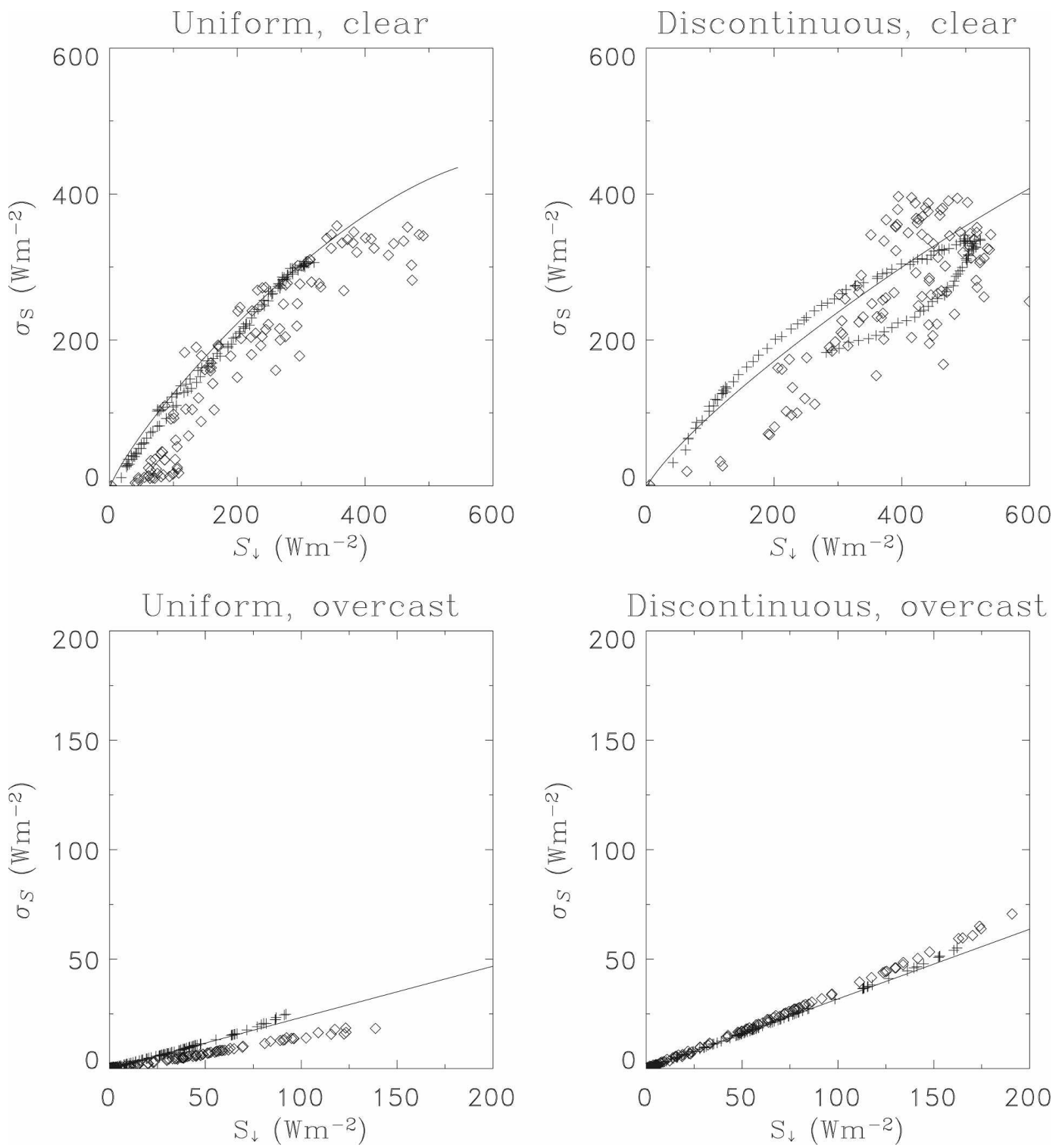

FIG. 13. Std dev plotted against average for subcanopy solar radiation in the (left) uniform and (right) discontinuous stands under (top) clear and (bottom) overcast conditions. Diamonds show measurements, crosses show simulations, and lines show parameterizations.

plicit model well but do not reproduce the clear-sky hysteresis for the discontinuous stand; hysteresis could be represented at the expense of replacing the constant parameters in Eq. (19) with functions of azimuth.

\section{Conclusions and discussion}

An orthophotograph and lidar elevations were used to map the location, height, and crown diameter of coniferous trees in an area of varying canopy density and structure. The resulting map, along with above-canopy measurements of incoming solar radiation and an esti- mation of the diffuse fraction, was used in a spatially explicit model of the transmission of direct and diffuse radiation to the surface beneath the canopy. Spatial statistics of simulated subcanopy radiation were compared with radiometer array measurements. The simulation was better for a discontinuous stand than for a uniform stand; discrepancies can largely be attributed to an overestimate of canopy cover in the denser areas.

Confidence intervals for sample statistics were estimated by repeated sampling of the model output; these turn out to be rather broad for 5-min averages with a 10 -radiometer array, but are greatly reduced when 


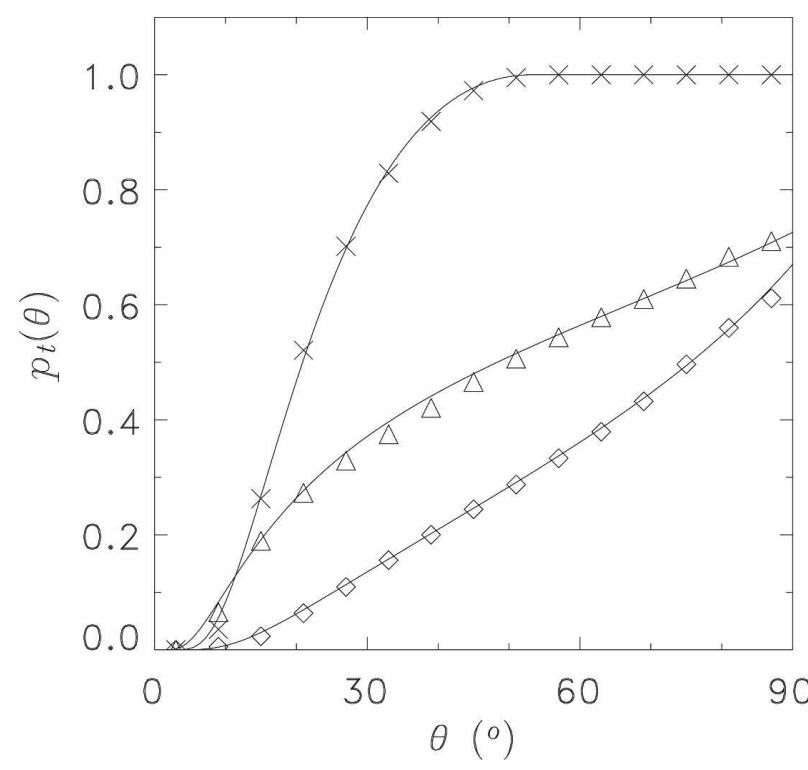

FIG. 14. Azimuthally averaged gap fractions for the uniform stand (diamonds), the discontinuous stand (triangles), and the clearing (crosses). The fitted lines are given by Eqs. (18) and (19) with parameters from Table 1 .

daily average statistics are considered. The standard deviation in subcanopy radiation decreases as the averaging period is increased, and decreases more rapidly for the uniform stand than the discontinuous stand.

Efficient parameterizations for the spatial average and standard deviation of subcanopy solar radiation were derived from the spatially explicit model. Five parameters were used in addition to above-canopy radiation and solar elevation inputs: the average and standard deviation of the sky-view factor under a stand, and three parameters fitted to the gap fraction. These were taken from model simulations, but could equally well be obtained with hemispherical photography. For application on larger scales, it may be possible to relate the parameters to more readily available canopy measures by applying the model to a wide range of canopy types. All of the parameters have physical meaning; the average and standard deviation of sky view are strongly

TABle 1. Parameter values fitting Eq. (18) to gap fractions and statistics of sky view used in parameterizations of radiation statistics.

\begin{tabular}{cccc}
\hline \hline & Uniform & Discontinuous & Clearing \\
\hline$a$ & -0.48 & -0.16 & 1.6 \\
$b$ & 1.2 & 0.48 & -0.96 \\
$c$ & 0.88 & 0.48 & -1.28 \\
$\bar{v}$ & 0.25 & 0.45 & 0.83 \\
$\sigma_{v}$ & 0.059 & 0.142 & 0.021 \\
\hline
\end{tabular}

related to the average and standard deviation of transmission for diffuse radiation, and the gap fraction parameters can be regarded as the first three terms in a Fourier expansion of the apparent foliage projection function.

In future work, we intend to extend the model to include thermal radiation and use it to investigate the spatial variation of snowmelt beneath forest canopies (Faria et al. 2000; Woo and Giesbrecht 2000). The model will be run with a range of real and synthetic stand maps and slopes to investigate how subcanopy radiation statistics scale with canopy characteristics and topography. Further consideration will be given to methods of mapping canopy structure using photography and lidar; as lidar may underestimate tree heights because of the probability of the laser beam penetrating some distance into the canopy, and the model simulates the probability of radiation penetrating the canopy, it may be possible to use the model to assimilate lidar data.

Acknowledgments. This work was supported by grants from NERC and NOAA. Datasets were supplied by CLPX through the National Snow and Ice Data Center.

\section{REFERENCES}

Battaglia, M. A., P. Mou, B. Palik, and R. J. Mitchell, 2002: The effect of spatially variable overstory on the understory light environment of an open-canopied longleaf pine forest. Can. J. For. Res., 32, 1984-1991.

Bunting, P., and R. Lucas, 2006: The delineation of tree crowns in Australian mixed species forests using hyperspectral Compact Airborne Spectorgraphic Imager (CASI) data. Remote Sens. Environ., 101, 230-248.

Chang, A. T. C., J. L. Foster, and D. K. Hall, 1996: Effects of forest on the snow parameters derived from microwave measurements during the BOREAS winter field campaign. $H y$ drol. Processes, 10, 1565-1574.

Chen, J. M., P. M. Rich, S. T. Gower, J. M. Norman, and S. Plummer, 1997: Leaf area index of boreal forests: Theory, techniques, and measurements. J. Geophys. Res., 102, 29429 29443.

Courbaud, B., F. de Coligny, and T. Cordonnier, 2003: Simulating radiation distribution in a heterogeneous Norway spruce forest on a slope. Agric. For. Meteor., 116, 1-18.

Culvenor, D. S., 2002: TIDA: An algorithm for the delineation of tree crowns in high spatial resolution remotely sensed imagery. Comput. Geosci., 28, 33-44.

Dozier, J., and J. Frew, 1990: Rapid calculation of terrain parameters for radiation modelling from digital elevation data. IEEE Trans. Geosci. Remote Sens., 28, 963-969.

Erbs, D. G., S. A. Klein, and J. A. Duffie, 1982: Estimation of the diffuse radiation fraction for hourly, daily and monthlyaverage global radiation. Sol. Atmos. Energy, 28, 293-302.

Essery, R. L. H., and J. W. Pomeroy, 2004: Implications of spatial distributions of snow mass and melt energy on snowcover 
depletion: Theoretical considerations. Ann. Glaciol., 38, 261265.

Faria, D., J. W. Pomeroy, and R. L. H. Essery, 2000: Effect of covariance between ablation and snow water equivalent on depletion of snow-covered area in a forest. Hydrol. Processes, 14, 2683-2695.

Frazer, G. W., C. D. Canham, and K. P. Lertzman, 1999: Gap Light Analyzer (GLA), Version 2.0: Users manual and program documentation. Simon Fraser University, Burnaby, BC, Canada, and the Institute of Ecosystem Studies, Millbrook, NY, 40 pp.

Gougeon, F. A., 1995: A crown-following approach to automatic delineation of individual tree crowns in high spatial resolution aerial images. Can. J. Remote Sens., 21, 274-284.

Hardy, J. P., R. Melloh, G. Koenig, D. Marks, A. Winstral, J. W. Pomeroy, and T. Link, 2004: Solar radiation transmission through conifer canopies. Agric. For. Meteor., 126, 257-270.

Kaufmann, M. R., C. B. Edminster, and C. A. Troendle, 1982: Leaf area determinations for subalpine tree species in the central Rocky Mountains. USDA Forest Service Research Paper RM-238, U.S. Department of Agriculture, 7 pp.

Klein, A., D. K. Hall, and G. A. Riggs, 1998: Improving snow cover mapping in forests through the use of a canopy reflectance model. Hydrol. Processes, 12, 1723-1744.

Li, X., A. H. Strahler, and C. E. Woodcock, 1995: A hybrid geometric optical-radiative transfer approach for modelling albedo and directional reflectance of discontinuous canopies. IEEE Trans. Geosci. Remote Sens., 33, 466-480.

Link, T. E., D. Marks, and J. P. Hardy, 2004: A deterministic method to characterize canopy radiative transfer properties. Hydrol. Processes, 18, 3583-3594.

Liu, J., R. A. Melloh, C. E. Woodcock, R. E. Davis, and E. S. Ochs, 2004: The effect of viewing geometry and topography on viewable gap fractions through forest canopies. Hydrol. Processes, 18, 3595-3607.

Magnussen, S., P. Eggermont, and V. N. LaRiccia, 1999: Recovering tree heights from airborne laser scanner data. For. Sci., 45, 407-422.

Melloh, R., J. Ballard, J. Hardy, C. Woodcock, J. Liu, J. Smith, G. Koenig, and R. Davis, 2003: Spatial distribution of canopy gaps in lodgepole pine forest. Proc. 60th Eastern Snow Conf., Sherbrooke, QC, Canada, Easter Snow Conference, 111-123.

Miller, S. L., 2003: CLPX-airborne: Infrared orthophotography and LiDAR topographic mapping. National Snow and Ice Data Center, Boulder, CO, digital media. [Available online at http://nsidc.org/data/docs/daac/nsidc0157_clpx_lidar/.]

Nijssen, B., and D. Lettenmaier, 1999: A simplified approach for predicting shortwave radiation transfer through boreal forest canopies. J. Geophys. Res., 104, 27 859-27 868.

Nilson, T., 1971: A theoretical analysis of the frequency of gaps in plant stands. Agric. Meteor., 8, 25-38.
Pinty, B., and Coauthors, 2004: Radiation Transfer Model Intercomparison (RAMI) exercise: Results from the second phase. J. Geophys. Res., 109, D06210, doi:10.1029/ 2003JD004252.

Pollock, R., 1996: The automatic recognition of individual trees in aerial images of forests based on a synthetic tree crown image model. Ph.D. thesis, University of British Columbia, 189 pp.

Pomeroy, J. W., and K. Dion, 1996: Winter radiation extinction and reflection in a boreal pine canopy: Measurements and modelling. Hydrol. Processes, 10, 1591-1608.

— A. Rowlands, J. Hardy, T. Link, D. Marks, R. Essery, J.-E. Sicart, and C. Ellis, 2008: Spatial variability of shortwave irradiance for snowmelt in forests. J. Hydrometeor., in press.

Price, M. A., 1997: A simplified scalable shading model for radiation at the forest floor. M.S. thesis, Dept. of Mathematics, Oregon State University, 43 pp.

Riaño, D., F. Valladares, S. Condés, and E. Chuvieco, 2004: Estimation of leaf area index and covered ground from airborne laser scanner (LiDAR) in two contrasting forests. Agric. For. Meteor., 124, 269-275.

Rich, P. M., 1990: Characterizing plant canopies with hemispherical photographs. Remote Sens. Rev., 5, 13-29.

Satterlund, D. R., 1983: Forest shadows: How much shelter in a shelterwood? For. Ecol. Manage., 5, 27-37.

Sellers, P. J., Y. Mintz, Y. C. Sud, and A. Dalcher, 1986: A simple biosphere model for use within general circulation models. $J$. Atmos. Sci., 43, 505-531.

Sheng, Y. W., P. Gong, and G. S. Biging, 2001: Model-based conifer-crown surface reconstruction from high-resolution aerial images. Photogramm. Eng. Remote Sens., 67, 957-965.

Song, C., and L. E. Band, 2004: MVP: A model to simulate the spatial patterns of photosynthetically active radiation under discrete forest canopies. Can. J. For. Res., 34, 1192-1203.

Stadt, K. J., V. J. Leiffers, R. J. Hall, and C. Messier, 2005: Spatially explicit modelling of PAR transmission and growth of Picea glauca and Abies balsamea in the boreal forests of Alberta and Quebec. Can. J. For. Res., 35, 1-12.

Strand, E., A. M. S. Smith, S. C. Bunting, L. A. Vierling, D. B. Hann, and P. E. Gessler, 2006: Wavelet estimation of vegetation spatial patterns in multitemporal aerial photography. Int J. Remote Sens., 27, 2049-2054.

Verseghy, D. L., N. A. McFarlane, and M. Lazare, 1993: CLASS A Canadian land surface scheme for GCMs, II. Vegetation model and coupled runs. Int. J. Climatol., 13, 347-370.

Woo, M.-K., and M. A. Giesbrecht, 2000: Simulation of snowmelt in a subarctic spruce woodland: 1 . Tree model. Water Resour. Res., 36, 2275-2285.

Yang, R., M. A. Friedl, and W. Ni, 2001: Parameterization of shortwave radiation fluxes for nonuniform vegetation canopies in land surface models. J. Geophys. Res., 106, $14275-$ 14286. 